\title{
Article \\ A G316A Polymorphism in the Ornithine Decarboxylase Gene Promoter Modulates MYCN-Driven Childhood Neuroblastoma
}

\author{
Laura D. Gamble ${ }^{1,+} \mathbb{D}^{\mathbb{D}}$, Stefania Purgato ${ }^{2, \dagger}$, Michelle J. Henderson ${ }^{1}$, Simone Di Giacomo ${ }^{2} \mathbb{D}$, \\ Amanda J. Russell ${ }^{3}{ }^{\circ}$, Paolo Pigini ${ }^{2}$, Jayne Murray ${ }^{1}$, Emanuele Valli ${ }^{1}$, Giorgio Milazzo ${ }^{2}{ }^{\circledR}$, Federico M. Giorgi ${ }^{2}{ }^{(0)}$, \\ Mark Cowley ${ }^{1}{ }^{\mathbb{D}}$, Lesley J. Ashton ${ }^{4}$, Jaydutt Bhalshankar ${ }^{5}$, Gudrun Schleiermacher ${ }^{5}{ }^{\circledR}$, Ali Rihani ${ }^{6}$, \\ Tom Van Maerken ${ }^{6}$, Jo Vandesompele ${ }^{6}{ }^{(\mathbb{D}}$, Frank Speleman ${ }^{6}$, Rogier Versteeg ${ }^{7}$, Jan Koster ${ }^{7}{ }^{\circledR}$, Angelika Eggert ${ }^{8}$, \\ Rosa Noguera ${ }^{9,10}{ }^{(\mathbb{D},}$, Raymond L. Stallings ${ }^{11}$, Gian Paolo Tonini ${ }^{12}$, Kwun Fong ${ }^{13}$, Zalman Vaksman ${ }^{14,15}$, \\ Sharon J. Diskin ${ }^{14,15}$, John M. Maris ${ }^{14,15}$, Wendy B. London ${ }^{16}$, Glenn M. Marshall 1,17, David S. Ziegler ${ }^{1,17}$, \\ Michael D. Hogarty ${ }^{14,15}\left[\right.$, , Giovanni Perini ${ }^{2,+}$, Murray D. Norris ${ }^{1,18,+}$ and Michelle Haber ${ }^{1, *,+}$
}

1 Children's Cancer Institute, Lowy Cancer Research Centre, UNSW Australia, PO Box 81, Randwick, NSW 2031, Australia; lgamble@ccia.org.au (L.D.G.); mhenderson@ccia.org.au (M.J.H.); jmurray@ccia.org.au (J.M.); evalli@ccia.org.au (E.V.); mcowley@ccia.org.au (M.C.); Glenn.Marshall@health.nsw.gov.au (G.M.M.); dziegler@ccia.org.au (D.S.Z.); MNorris@ccia.unsw.edu.au (M.D.N.)

2 Department of Pharmacy and Biotechnology, University of Bologna, 40126 Bologna, Italy; stefania.purgato2@unibo.it (S.P.); simone.digiacomo2@unibo.it (S.D.G.); paolo.pigini2@unibo.it (P.P.); giorgio.milazzo2@unibo.it (G.M.); federico.giorgi@unibo.it (F.M.G.); giovanni.perini@unibo.it (G.P.)

check for

updates

Citation: Gamble, L.D.; Purgato, S.; Henderson, M.J.; Di Giacomo, S.; Russell, A.J.; Pigini, P.; Murray, J.; Valli, E.; Milazzo, G.; Giorgi, F.M.; et al. A G316A Polymorphism in the Ornithine Decarboxylase Gene Promoter Modulates MYCN-Driven Childhood Neuroblastoma. Cancers 2021, 13, 1807. https://doi.org/ $10.3390 /$ cancers 13081807

Academic Editor:

Vladimir Spiegelman

Received: 7 March 2021

Accepted: 6 April 2021

Published: 9 April 2021

Publisher's Note: MDPI stays neutral with regard to jurisdictional claims in published maps and institutional affiliations.

Copyright: (c) 2021 by the authors. Licensee MDPI, Basel, Switzerland. This article is an open access article distributed under the terms and conditions of the Creative Commons Attribution (CC BY) license (https:/ / creativecommons.org/licenses/by/ $4.0 /)$.
3 Cancer Research Program, The Garvan Institute of Medical Research, Darlinghurst, NSW 2010, Australia; a.russell@garvan.org.au

4 Research Portfolio, University of Sydney, Sydney, NSW 2008, Australia; lesley.ashton@sydney.edu.au

5 SIREDO, Department of Paediatric, Adolescents and Young Adults Oncology and INSERM U830, Institut Curie, 26 rue d'Ulm, 75005 Paris, France; jaydutt.bhalshankar@curie.fr (J.B.); gudrun.schleiermacher@curie.fr (G.S.)

6 Center for Medical Genetics, Ghent University, C. Heymanslaan 10, 9000 Ghent, Belgium; ali.rihani@ki.se (A.R.); Tom.VanMaerken@UGent.be (T.V.M.); jo.vandesompele@ugent.be (J.V.); franki.speleman@ugent.be (F.S.)

7 Department of Oncogenomics, Academic Medical Center, University of Amsterdam, 1100 Amsterdam, The Netherlands; r.versteeg@amc.uva.nl (R.V.); jankoster@amsterdamumc.nl (J.K.)

8 Department of Pediatric Hematology, Oncology and SCT, Charité-University Hospital Berlin, Campus Virchow-Klinikum, 10117 Berlin, Germany; angelika.eggert@charite.de

9 Department of Pathology, Medical School, University of Valencia, 46010 Valencia, Spain; Rosa.Noguera@uv.es

10 CIBERONC-INCLIVA, Biomedical Health Research Institute, 46010 Valencia, Spain

11 Molecular and Cellular Therapeutics, Royal College of Surgeons in Ireland, D02 YN77 Dublin 2, Ireland; rstallings@rcsi.ie

12 Neuroblastoma Laboratory, Fondazione Istituto di Ricerca Pediatrica Città della Speranza, 35127 Padova, Italy; gp.tonini@irpcds.org

13 Thoracic Research Centre, University of Queensland, The Prince Charles Hospital, Brisbane, QLD 4032, Australia; fongk@health.qld.gov.au

14 Division of Oncology and Center for Childhood Cancer Research, Children's Hospital of Philadelphia, Philadelphia, PA 19104, USA; vaksmanz@email.chop.edu (Z.V.); diskin@email.chop.edu (S.J.D.); Maris@email.chop.edu (J.M.M.); hogartym@email.chop.edu (M.D.H.)

15 Department of Pediatrics, Perelman School of Medicine, University of Pennsylvania, Philadelphia, PA 19104, USA

16 Dana-Farber/Boston Children's Cancer and Blood Disorders Center, Harvard Medical School, Boston, MA 02215, USA; wendy.london@childrens.harvard.edu

17 Kids Cancer Centre, Sydney Children's Hospital, High St, Randwick, NSW 2031, Australia

18 Centre for Childhood Cancer Research, University of New South Wales, Sydney, NSW 2052, Australia

* Correspondence: mhaber@ccia.unsw.edu.au; Tel.: +61-(02)-9385-2170

+ These authors contributed equally to this work.

Simple Summary: Neuroblastoma is a devasting childhood cancer in which multiple copies (amplification) of the cancer-causing gene MYCN strongly predict poor outcome. Neuroblastomas are reliant on high levels of cellular components called polyamines for their growth and malignant behavior, and the gene regulating polyamine synthesis is called ODC1. ODC1 is often coamplified with MYCN, 
and in fact is regulated by MYCN, and like MYCN is prognostic of poor outcome. Here we studied a naturally occurring genetic variant or polymorphism that occurs in the ODC1 gene, and used gene editing to demonstrate the functional importance of this variant in terms of ODC1 levels and growth of neuroblastoma cells. We showed that this variant impacts the ability of MYCN to regulate ODC1, and that it also influences outcome in neuroblastoma, with the rarer variant associated with a better survival. This study addresses the important topic of genetic polymorphisms in cancer.

Abstract: Ornithine decarboxylase (ODC1), a critical regulatory enzyme in polyamine biosynthesis, is a direct transcriptional target of $\mathrm{MYCN}$, amplification of which is a powerful marker of aggressive neuroblastoma. A single nucleotide polymorphism (SNP), G316A, within the first intron of ODC1, results in genotypes wildtype GG, and variants AG/AA. CRISPR-cas9 technology was used to investigate the effects of AG clones from wildtype $M Y C N$-amplified SK-N-BE(2)-C cells and the effect of the SNP on MYCN binding, and promoter activity was investigated using EMSA and luciferase assays. AG clones exhibited decreased ODC1 expression, growth rates, and histone acetylation and increased sensitivity to ODC1 inhibition. MYCN was a stronger transcriptional regulator of the $O D C 1$ promoter containing the $\mathrm{G}$ allele, and preferentially bound the $\mathrm{G}$ allele over the A. Two neuroblastoma cohorts were used to investigate the clinical impact of the SNP. In the study cohort, the minor AA genotype was associated with improved survival, while poor prognosis was associated with the GG genotype and AG/GG genotypes in MYCN-amplified and non-amplified patients, respectively. These effects were lost in the GWAS cohort. We have demonstrated that the ODC1 G316A polymorphism has functional significance in neuroblastoma and is subject to allele-specific regulation by the MYCN oncoprotein.

Keywords: ODC1; MYCN; SNP; neuroblastoma

\section{Introduction}

Neuroblastoma is a childhood cancer that often presents as high-risk disease that is resistant to treatment. Amplification of the $M Y C N$ oncogene occurs in $20-25 \%$ of cases, and is a powerful and reliable marker of poor prognosis [1]. MYCN belongs to the MYC family of transcription factors and functions by forming heterodimers with MAX that typically bind six nucleotide 'E-box' sequences at the promoters of target genes.

Polyamines are organic cations that play critical roles in a number of cellular processes and abnormalities in the control of polyamine metabolism and transport can result in increased polyamine levels promoting tissue proliferation and tumor formation [2]. Ornithine decarboxylase (ODC1) is a critical regulatory enzyme in the polyamine biosynthesis pathway and is a direct transcriptional target of MYC oncoproteins [3,4]. It is the first enzyme in polyamine synthesis and is the rate limiting step for the conversion of ornithine to the primary polyamine, putrescine. ODC1 is essential for normal development and tissue repair in mammals but is downregulated in most adult tissues [5].

ODC1 has been implicated as an important gene during the early stages of tumor progression and is highly expressed in a variety of cancer types [6-10]. High ODC1 expression is found in MYCN-amplified neuroblastoma and is associated with a poor prognosis [10,11]. However, high ODC1 expression also predicts poor outcome in MYCN non-amplified neuroblastoma suggesting it has oncogenic abilities independent of MYCN [10,11]. Disabling ODC1 using the specific inhibitor difluoromethylornithine (DFMO) inhibits neuroblastoma proliferation in vitro as well as in transgenic and patient derived xenograft mouse models of neuroblastoma [10-13] and based on these findings, clinical trials are being conducted combining DFMO with conventional chemotherapy and/or chemoimmunotherapy in relapsed/refractory neuroblastoma patients (ClinicalTrials.gov Identifier: NCT02030964 and NCT03794349).

A single nucleotide polymorphism (SNP, rs2302615) in the regulatory region of ODC1, located in the proximal region of intron $1+316$ nucleotides $3^{\prime}$ of the transcriptional start 
site (chr2(hg19):g.10588138C > T) has been identified (Figure S1) [14]. This SNP, G316A, is located between neighboring E-boxes that cooperate to influence ODC1 promoter activity $[3,14,15]$, while the sequences flanking these E-boxes have been shown to influence binding of MYC $[16,17]$. Three possible genotypes result from the SNP: GG, AG and the variant AA which occurs at a low frequency of $7-10 \%$ in the general population $[14,18]$. A previous study has shown that the transcriptional repressor Mad1 suppresses the activity of the $O D C 1$ promoter in colon cancer cells containing the A-allele but not the G-allele, and other studies have also reported that the E-box repressors MAD4 (MXD4) and MXI1 bind less strongly in cells homozygous for the GG genotype [18,19]. These findings suggest allele-specific regulation of ODC1 by E-box transcription factors. It has also been shown that the GG genotype is associated with improved survival compared to people with AG/AA genotypes in colorectal and breast cancer patients $[18,19]$. This finding contrasts with those from a colon cancer prevention trial, in which individuals with the AA genotype who reported using aspirin were $10 \times$ less likely to have an adenoma recurrence compared to non-aspirin users with the GG genotype [20,21].

In this study, we investigated both the functional and prognostic significance of the G316A SNP in neuroblastoma cell lines and tumors. We demonstrate that the ODC1 G316A polymorphism influences ODC1 expression, neuroblastoma cell growth and response to DFMO. In contrast to studies in adult cancer patients the $G$ allele was associated with a poorer outcome in neuroblastoma patients. In addition, $\mathrm{MYCN}$ preferentially binds the $\mathrm{G}$ allele over the A allele, and produces a greater stimulatory effect on the ODC1 promoter in the presence of the $\mathrm{G}$ allele.

\section{Results}

2.1. The 316A Allele Is Associated with Reduced Cell Growth and ODC1 Expression, and Increased Sensitivity to DFMO in Cas9-Edited Neuroblastoma Cells

We utilized CRISPR-Cas9 technology to examine the functional effects of the ODC1 SNP genotype in $M Y C N$-amplified SK-N-BE(2)-C cells exhibiting the GG genotype. Adopting the HDR-mediated editing strategy (Figure 1A) and donor DNA from neuroblastoma NBL-S cells, which are of AG genotype, we obtained two independent cell lines carrying the AG genotype (AG-1 and AG-2, Figure S2). Both ODC1 mRNA and protein expression were markedly decreased in AG-1 and AG-2 lines when compared to wildtype cells (Figure 1B,C), and they also had a significantly reduced proliferation rate following 8 days of growth (Figure 1D). Using colony assays, the AG lines were found to be more sensitive to the ODC1 inhibitor DFMO (Figure 1E). Together these observations suggest that the A allele variant reduces $O D C 1$ transcription, thereby moderating neuroblastoma cell proliferation and influencing response to DFMO-based therapies. Chromatin immunoprecipitation (ChIP) experiments were performed to determine the acetylation status in the region spanning the SNP (construct shown in Figure S3A). Consistent with the expression results, $\mathrm{H} 3$ histone acetylation in this region was decreased in AG clones compared to the parental cells $(p<0.005)$, (Figure 1F), demonstrating an important role of the G316A SNP in influencing the chromatin status of the $O D C 1$ promoter region. Given the decreased level of histone acetylation in the AG clones, we next explored whether decreased MYCN activity at this locus is also associated with the A allele. 


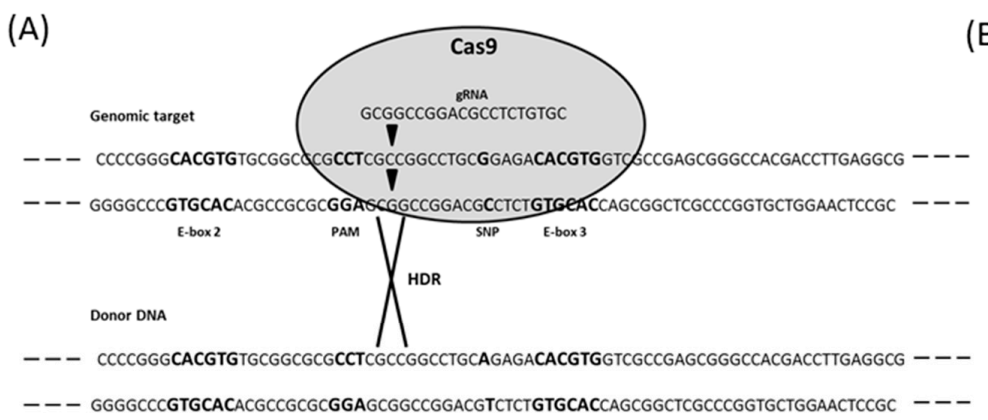

(C)

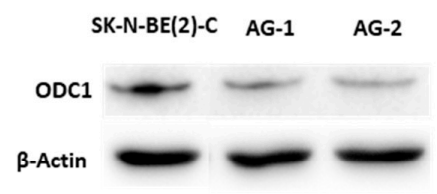

(D)

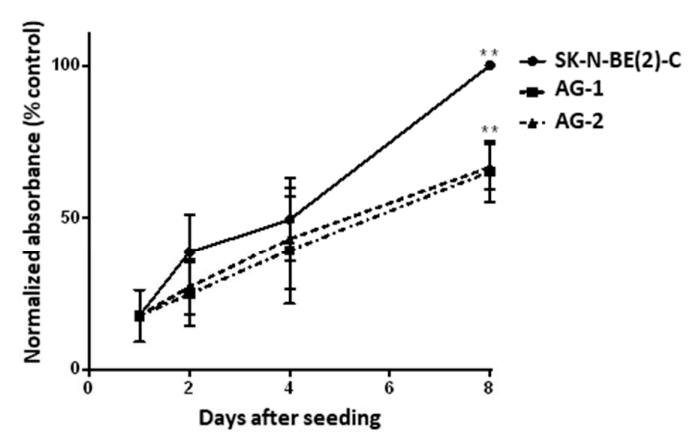

(E)

(F)

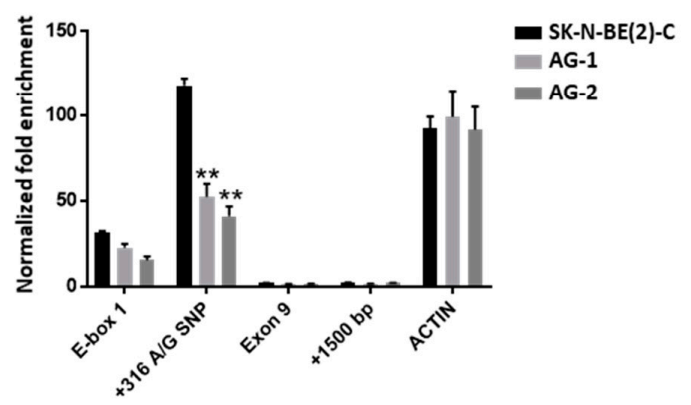

(B)
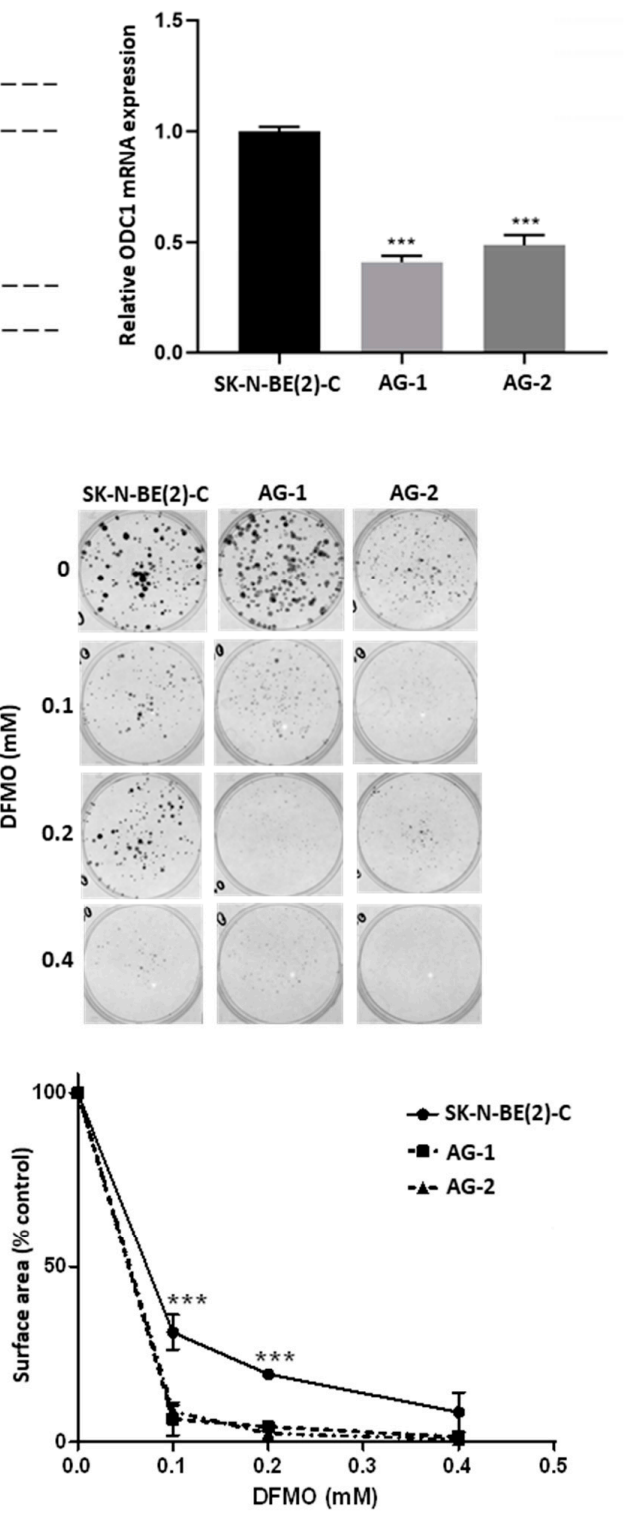

Figure 1. Analysis of AG clones generated from the GG SK-N-BE(2)-C cell line by CRISPR-Cas9 editing. (A) The CRISPRCas9 editing strategy was adopted to edit SK-N-BE(2)-C cells. The gRNA cleavage site (inverted triangles) is located $9 \mathrm{bp}$ upstream of the G316A SNP. The donor DNA sequence carries the A SNP mutation (black). (B,C) ODC1 expression analysis by qRT-PCR (B) and Western blot (C) of CRISPR-edited clones AG-1 and AG-2, compared to parental SK-N-BE(2)-C cells. qRT-PCR data were normalized using GUSB as reference gene, and standardized as previously described [22]. B-actin was used as the loading control for the Western blot. Uncropped WB images are available in Figure S10. (D) Proliferation rates of CRISPR-edited clones. Cell growth was measured by BrdU assay. Absorbance data were normalized to day 1 and SK-N-BE(2)-C was used as the control. (E) Analysis of the effect of DFMO on CRISPR-edited clones by clonogenic assay. The total area occupied by cell colonies was measured by crystal violet staining and subsequent ImageJ analysis. All $p$ values were determined by one-way ANOVA tests and compared to SK-N-BE(2)-C as control. (F) ChIP analysis of H3-histone acetylation of the G316A SNP spanning region in the CRISPR-edited clones. The analyzed sequences in the ODC1 locus are E-box1, G316A SNP, Exon 9 and a sequence located +1500 bp downstream of the locus. Acetylation of a region in the Actin gene was used as a control. Enrichment data were normalized to a non-acetylated region located 15,000 bp upstream of the ODC1 locus. A representation of the analyzed region is shown in Figure S3A. ${ }^{* *} p<0.005,{ }^{* * *} p<0.001$. All experiments were performed at least 3 times. 


\subsection{The 316 A Allele Influences ODC1 Promoter Activity In Vitro}

Electrophoretic mobility shift assays (EMSA) were performed to determine the influence of the SNP genotype on the MYCN/MAX binding properties in the E-box sequence nearest to the polymorphism (E-box 3). Nuclear extracts from SK-N-BE(2)-C and Tet21N cells were incubated with radiolabeled DNA probes containing either the A or the G SNP plus the downstream E-box (Figure S3B). A stronger band was observed for the $G$ probe versus the A probe in the presence of nuclear extracts for both cell lines (Figure 2A,B, lanes 3 and 4), indicating weaker binding of MYCN/MAX to the A probe. This provides further evidence that the A SNP negatively influences ODC1 expression through reduced binding of the MYCN transcription factor and is consistent with previous data of ours showing that enrichment within the G316A SNP region following MYCN ChIP is approximately two-fold higher in SK-N-BE(2)-C cells, which we have determined to be of GG genotype, than in LAN1 cells, which are of AG genotype [13].

(A)

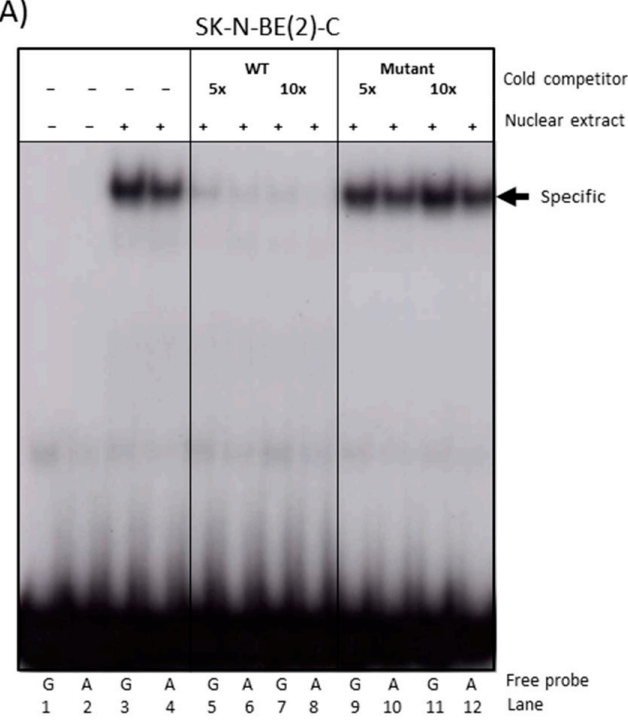

(B)

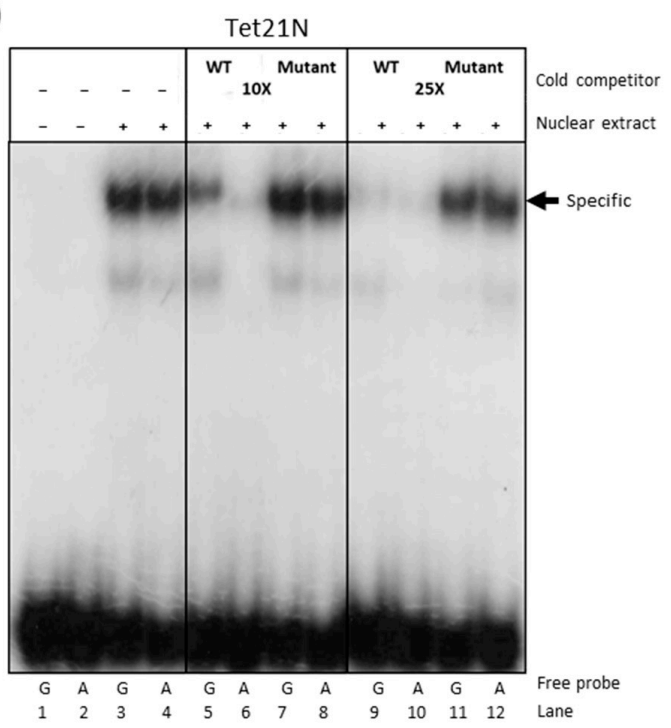

(C)

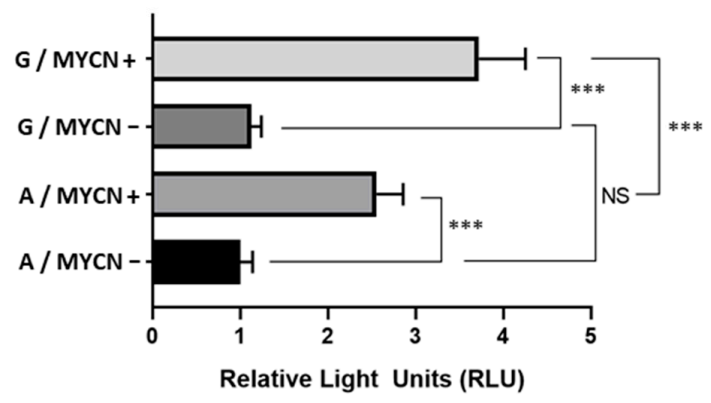

Figure 2. The effect of the ODC1 SNP on MYCN binding and ODC1 promoter activity. (A,B) MYCN binding was assessed using EMSA with SK-N-BE(2)-C (A) and Tet21N (B) nuclear extracts. Nuclear extracts were incubated in the presence of radiolabeled probes containing either the G SNP (G probe) or the A SNP (A probe), and E-box 3. Competition analysis was performed by incubating the binding reaction with 5-10-fold and 10-25-fold molar excess of unlabeled G probe carrying either a wild type (WT) or Mutant E-box. Quantification of the specific bands (arrow) is shown in Figure S4. Note that the order of the lanes differs between A and B. (C) Luciferase reporter assays in Tet21N cells transfected with a reporter plasmid containing a region of the ODC1 promoter that includes either the G or the A SNP. Untreated Tet21N cells with MYCN overexpression (MYCN+) were compared to tetracycline-treated cells (MYCN-), where MYCN overexpression is blocked. Replicates were standardized as previously described [22]. A two-way ANOVA was used to test for an interaction between the effects of the SNP and MYCN expression on promoter activity (SNP: $\mathrm{F}_{1,16}=20.0, p<0.001$; MYCN expression: $\mathrm{F}_{1,16}=203.4, p<0.001$; Interaction: $\mathrm{F}_{1,16}=13.0, p=0.002$ ) followed by Tukey's post-hoc test for multiple pairwise comparisons. A representation of all the constructs utilized for these experiments are shown in Figure S3B. ${ }^{* * *} p<0.001$. 
To specifically demonstrate that the bands for the A or the G SNP probes were associated with the MYCN/MAX complex, a competition assay using increasing amounts of cold wild-type or mutated probes for MYCN/MAX was performed. A reduction in binding of the MYCN/MAX complex to the A probe (shown by quantification of band intensity in Figure S4) was more pronounced than that observed with the $G$ probe, demonstrating that MYCN/MAX has higher affinity for the G SNP probe compared to the A probe. This is clearly demonstrated with the lower concentration of the cold wild-type probe in lanes 5 and 6 (Figures 2 and S4). As a negative control, a competition assay was performed using the same probe but containing a mutated canonical E-box and no reductions in band intensity were observed.

The minimal $+257 /+330$ DNA region of the ODC1 promoter was cloned upstream of a luciferase reporter cassette (Figure S3B) and the resultant vector was transfected into MYCN inducible Tet21N cells. We assessed the influence of the SNP on MYCN-mediated transactivation of $O D C 1$. There was a statistically significant interaction between the effects of the SNP and MYCN expression on promoter activity $(\mathrm{F}(1,16)=13.0, p=0.002)$. Tukey's post hoc tests showed that the promoter activity was strongly enhanced in MYCN-induced cells compared to non-induced cells regardless of genotype. However, consistent with previous findings, $O D C 1$ promoter activity was significantly reduced in constructs containing the A allele compared to those containing the G allele, following MYCN induction $(p<0.001$; Figure 2C). Comparing the stimulatory effect of MYCN on ODC1 promoters containing either allele, there was a $2.9+/-0.2$-fold increase in luciferase activity for the A allele, and a $3.9+/-0.2$-fold increase for the G allele suggesting that MYCN stimulates significantly increased levels of $O D C 1$ promoter activity in the presence of the G allele compared to the A allele $(p=0.013)$.

\subsection{The ODC1 316 A Allele and Outcome in Neuroblastoma Patients}

Our study cohort consisted of 839 primary neuroblastoma patient samples recruited from Europe, the USA and Australia prior to 2012, with the majority being recruited much earlier than this. The GWAS cohort from the USA consisted of neuroblastoma patient samples recruited up to 2016. In the study cohort, the AA genotype was found in 61 patients $(7.3 \%)$, the AG genotype in 272 patients $(32.4 \%)$, and the GG genotype in 506 patients $(60.3 \%)$, consistent with population distributions of the three genotypes previously reported (Table S1) $[14,18]$. The GWAS cohort consisted of 425 patients $(8.7 \%)$ with AA genotypes, 1963 patients (40.1\%) with AG genotypes and 2504 patients (51.2\%) with GG genotypes (Table S1). There was an association between tumour stage and genotype $(p=0.049)$, but no association between genotype and age or MYCN status in the study cohort. A weak association $(p=0.033)$ between $M Y C N$ amplification status and genotype was observed in the GWAS cohort (Table 1). As expected for a representative neuroblastoma cohort, stage/risk group, age and $M Y C N$ status were each prognostic of outcome in both cohorts $(p<0.001$, Figure S5). 
Table 1. The clinical and molecular characteristics of the 839 patients of the study cohort, and the 4892 patients of the GWAS cohort. In the study cohort, age was available for 638 patients, stage was available for 799 patients and MYCN amplification status was available for 837 patients. In the GWAS cohort, age was available for all patients, risk group was available for 4787 patients and $M Y C N$ amplification status was available for 4539 patients.

\begin{tabular}{|c|c|c|c|c|c|c|c|}
\hline & \multirow{2}{*}{ Factors } & & \multicolumn{3}{|c|}{ Genotype } & \multirow{2}{*}{ Total } & \multirow{2}{*}{$p$} \\
\hline & & & $\mathbf{A A}$ & $\mathbf{A G}$ & GG & & \\
\hline \multirow{6}{*}{ Study cohort } & \multirow{2}{*}{ Age } & $\leq 18$ months & $36(7.9 \%)$ & $141(31.0 \%)$ & $278(61.1 \%)$ & 455 & \multirow[t]{2}{*}{0.551} \\
\hline & & $>18$ months & $25(6.5 \%)$ & $130(33.9 \%)$ & $228(59.5 \%)$ & 383 & \\
\hline & \multirow{2}{*}{ Tumor stage } & Favourable & $31(8.9 \%)$ & $96(27.5 \%)$ & $223(63.7 \%)$ & 350 & \multirow[t]{2}{*}{0.049} \\
\hline & & Unfavourable & $28(6.2 \%)$ & $158(35.2 \%)$ & $263(58.6 \%)$ & 449 & \\
\hline & \multirow{2}{*}{$M Y C N$ status } & Non-amplified & $50(7.2 \%)$ & $224(32.2 \%)$ & $421(60.6 \%)$ & 695 & \multirow[t]{2}{*}{0.970} \\
\hline & & Amplified & $11(7.7 \%)$ & $45(31.7 \%)$ & $86(60.6 \%)$ & 142 & \\
\hline \multirow{7}{*}{ GWAS cohort } & \multirow{2}{*}{ Age } & $\leq 18$ months & $187(8.3 \%)$ & 887 (39.4\%) & 1177 (52.3\%) & 2251 & \multirow[t]{2}{*}{0.328} \\
\hline & & $>18$ months & $238(9.0 \%)$ & $1076(40.7 \%)$ & $1327(50.2 \%)$ & 2641 & \\
\hline & \multirow{3}{*}{ Risk group } & Low & $144(9.2 \%)$ & $622(39.9 \%)$ & 794 (50.9\%) & 1560 & \multirow[t]{3}{*}{0.826} \\
\hline & & Intermediate & $100(8.8 \%)$ & $451(39.9 \%)$ & $580(51.3 \%)$ & 1131 & \\
\hline & & High & $171(8.2 \%)$ & $855(40.8 \%)$ & $1070(51.0 \%)$ & 2096 & \\
\hline & \multirow{2}{*}{$M Y C N$ status } & Non-amplified & $341(9.2 \%)$ & 1469 (39.7\%) & $1889(51.1 \%)$ & 3699 & \multirow[t]{2}{*}{0.033} \\
\hline & & Amplified & $54(6.4 \%)$ & $349(41.5 \%)$ & $437(52.0 \%)$ & 840 & \\
\hline
\end{tabular}

Survival analysis showed improved EFS in the study cohort of neuroblastoma patients with tumors of AA genotype, although this trend did not achieve statistical significance (Table 2; Figure 3A and Figure S6A). Since ODC1 is an established MYC target gene [3,4], we investigated the possibility of differential effects of the SNP in subsets of patients depending on the MYCN status of their tumor. For patients with $M Y C N$ amplification, the presence of at least one A allele predicted a better outcome than GG homozygous patients ( $p=0.001,5$-year EFS of $41.1 \%$ for AA / AG compared to $22.4 \%$ for GG (Figure 3A, Table 2); $p=0.003,5$-year OS of $48.2 \%$ for AA/AG compared to $28.2 \%$ for GG (Figure S6A, Table 2)) and this remained significant following adjustment for age and stage (EFS: $p=0.003$, OS: $p=0.006$; Table S2). In patients without MYCN amplification, the AA genotype conferred a significantly better EFS, and a trend towards improved OS by comparison with tumors exhibiting an AG/GG genotype (Figure 3A and Figure S6A). These trends were verified in the three separate cohorts that make up the study cohort (Figure S7). Thus, in our study cohort, the impact of the G316A SNP appears to be influenced by MYCN and the adverse effect on outcome conferred by very high levels of MYCN oncoprotein in amplified tumors can be ameliorated by the presence of an A allele. However, in the GWAS cohort, which contains more recent patients who have undergone intensified therapy resulting in improved survival rates of high-risk patients compared to the study cohort (Figure S8), no significant associations between genotype and survival were observed regardless of MYCN amplification status (Figures 3B and S6B, Table 2). 
Table 2. Univariate Cox regression analysis of ODC SNP genotype groupings in neuroblastoma patients of the study cohort (EFS available for 839 neuroblastoma patients, and OS for 838), and the GWAS cohort (EFS and OS available for all patients).

\begin{tabular}{|c|c|c|c|c|c|}
\hline \multirow[b]{2}{*}{ Sample } & \multirow[b]{2}{*}{ Genotype } & \multicolumn{2}{|c|}{ Event-Free Survival } & \multicolumn{2}{|c|}{ Overall Survival } \\
\hline & & $\begin{array}{l}\text { Relative Hazard } \\
\qquad(95 \% \text { CI })\end{array}$ & $p$ & $\begin{array}{l}\text { Relative Hazard } \\
(95 \% \mathrm{CI})\end{array}$ & $p$ \\
\hline \multicolumn{6}{|c|}{ Study cohort } \\
\hline \multirow{2}{*}{ All patients } & GG & $0.98(0.77-1.24)$ & 0.847 & $0.99(0.75-1.29)$ & 0.914 \\
\hline & AG/GG & $1.63(0.95-2.78)$ & 0.074 & $1.45(0.81-2.59)$ & 0.212 \\
\hline \multirow{2}{*}{$\begin{array}{c}\text { MYCN } \\
\text { non-amplified }\end{array}$} & GG & $0.79(0.59-1.06)$ & 0.120 & $0.76(0.54-1.07)$ & 0.115 \\
\hline & $\mathrm{AG} / \mathrm{GG}$ & $2.46(1.09-5.45)$ & 0.025 & $2.05(0.84-5.01)$ & 0.103 \\
\hline \multirow{2}{*}{ MYCN amplified } & GG & $1.93(1.27-2.93)$ & 0.001 & $1.91(1.22-2.97)$ & 0.003 \\
\hline & $\mathrm{AG} / \mathrm{GG}$ & $1.07(0.52-2.21)$ & 0.846 & $1.13(0.52-2.45)$ & 0.752 \\
\hline \multicolumn{6}{|c|}{ GWAS cohort } \\
\hline \multirow{2}{*}{ All patients } & GG & $0.96(0.87-1.07)$ & 0.469 & $0.98(0.87-1.11)$ & 0.792 \\
\hline & AG/GG & $1.10(0.91-1.32)$ & 0.323 & $1.14(0.92-1.43)$ & 0.237 \\
\hline \multirow{2}{*}{$\begin{array}{c}\text { MYCN } \\
\text { non-amplified }\end{array}$} & GG & $0.88(0.77-1.01)$ & 0.063 & $0.87(0.74-1.03)$ & 0.107 \\
\hline & AG/GG & $1.02(0.81-1.29)$ & 0.858 & $1.03(0.78-1.37)$ & 0.828 \\
\hline \multirow{2}{*}{ MYCN amplified } & GG & $1.13(0.94-1.36)$ & 0.197 & $1.15(0.95-1.40)$ & 0.152 \\
\hline & AG/GG & $0.93(0.65-1.34)$ & 0.711 & $1.12(0.74-1.69)$ & 0.597 \\
\hline
\end{tabular}

Full multivariate analysis is shown in Table S2.

(A)

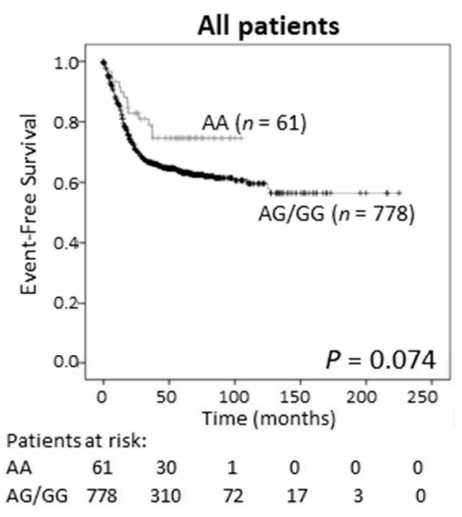

(B)

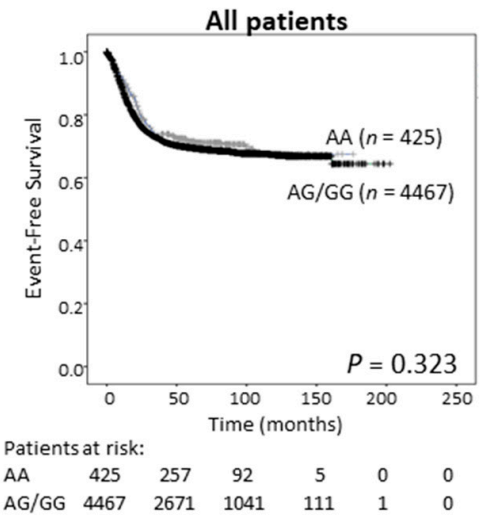

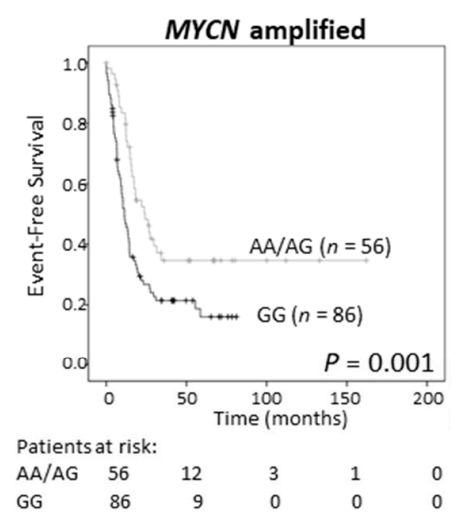

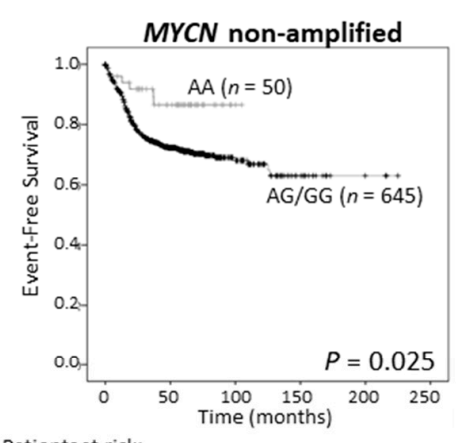

Patients at risk:
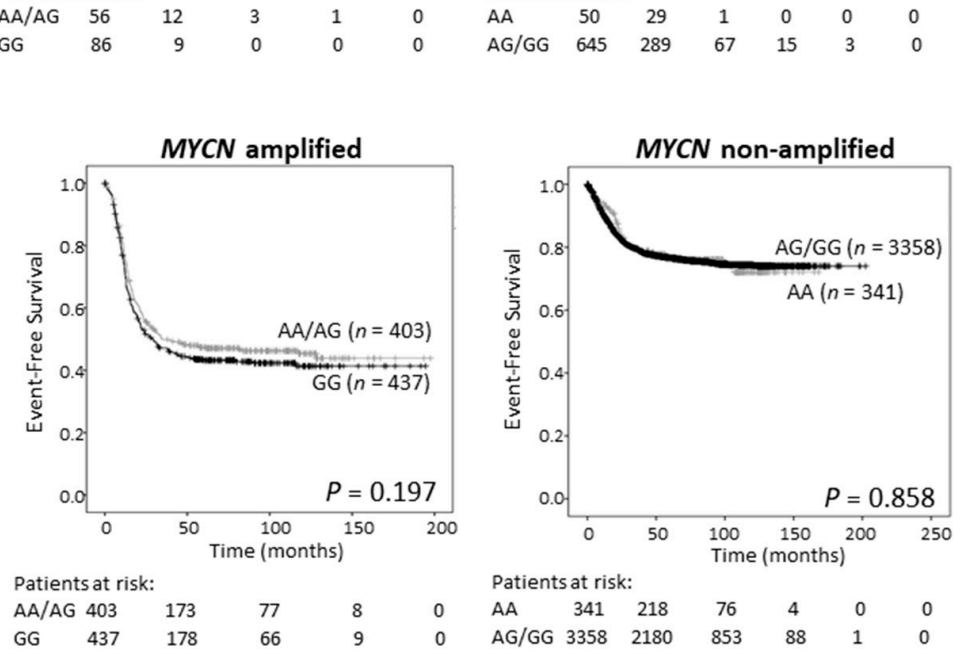

Figure 3. Event-free survival for the 839 neuroblastoma sample study cohort (A), and the 4892 patient GWAS cohort (B), grouped by genotype (AA vs. AA/AG for all patients and non-MYCN amplified patients, and AA/AG vs. GG for MYCN amplified patients). 


\subsection{A Divergent Role for ODC1 in Adult Cancers}

Earlier studies on breast and colorectal cancers reported that the G316A GG genotype is associated with a more favorable outcome than the AG/AA genotypes $[18,19]$. We investigated the association between SNP genotype and survival in a publicly available colorectal cancer cohort of 290 cases (Sieber, R2 Genomics Analysis and Visualization platform; https://hgserver1.amc.nl/cgi-bin/r2/main.cgi, accessed on 3 August 2020). We found that low, rather than high, expression of ODC1 mRNA was associated with poor EFS ( $p=0.027$, Figure 4A). We also examined a cohort consisting of 63 tumors of rectal origin, since an association between SNP genotype and outcome had previously been reported in this subtype [18]. Despite very few events, there was a trend towards improved survival in patients with the GG genotype compared to patients with AA/AG genotypes (Figure 4B). These findings contrast to the observed association between poor neuroblastoma outcome and high ODC1 expression, suggesting a different role for ODC1 in colorectal cancer compared with neuroblastoma.

(A)

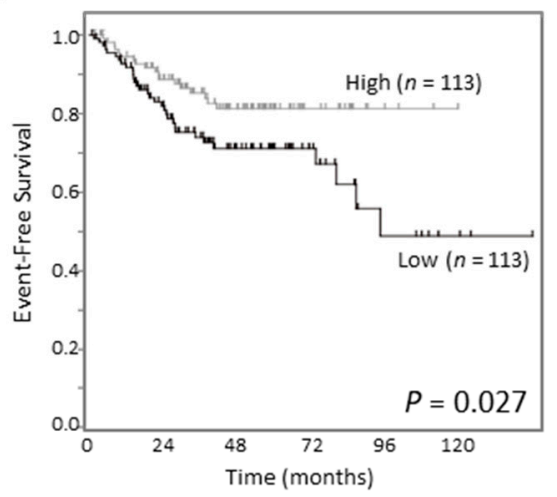

(C)

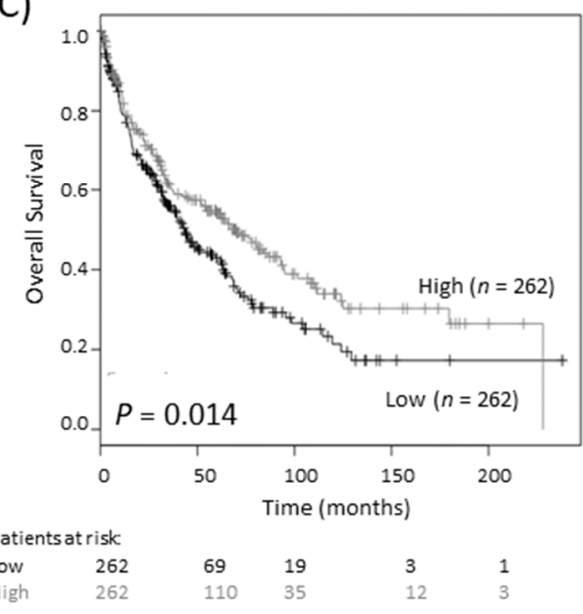

(B)

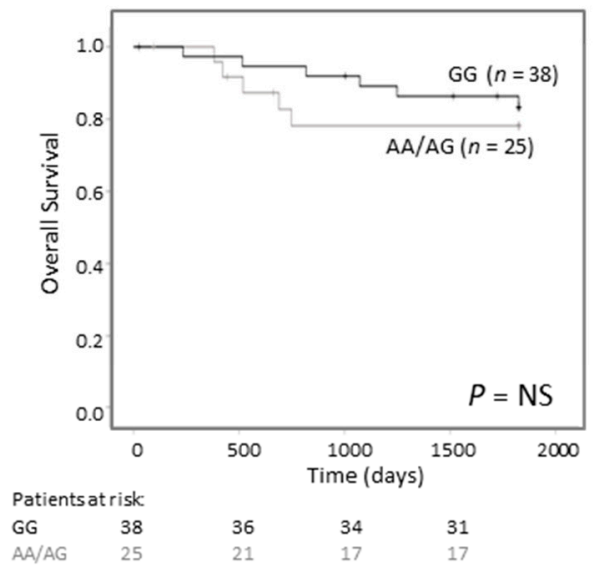

(D)

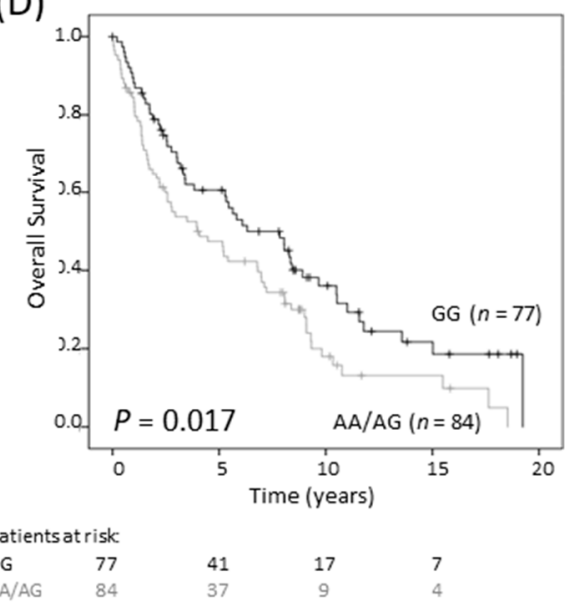

Figure 4. G316A ODC1 promoter SNP and its prognostic significance in colorectal and lung cancer. (A) In an online colorectal cancer dataset of 226 primary samples (Sieber dataset), low expression of ODC1 was prognostic of poor outcome (dichotomized at the median). This dataset is available on the R2 platform (R2: Genomics Analysis and Visualization Platform, https:/ /hgserver1.amc.nl/cgi-bin/r2/main.cgi, accessed on 3 August 2020). Patients at risk are not shown as they cannot be generated using this platform. (B) In a cohort of 63 rectal cancer patients, where most patients had a favorable outcome, the AA/AG genotype tended to have a poorer overall outcome than the GG genotype. NS-not significant. (C) In a lung cancer cohort of 524 squamous cell carcinoma patients, low ODC1 expression is associated with worse outcome. Data was obtained from KM Plotter [23]. (D) In a second cohort of 161 squamous cell carcinomas patients, those with AG/AA genotypes tended to have a worse outcome compared with GG genotypes. 
We obtained a similar result in lung cancer patients with small cell carcinoma. Firstly, in a publicly available dataset of 524 patients, low levels of ODC1 mRNA expression were associated with shorter overall survival (Figure 4C). In a cohort of 366 patients with non-small cell lung cancer (NSCLC) of mixed histologies, poorer survival was observed in patients with at least one A allele compared to the GG genotype ( $p=0.039$, Table S3). This relationship was found to be strongest in SCC ( $p=0.017$, Figure 4D) and was maintained following adjustment for ECOG status, advanced tumor stage and age ( $p=0.049$, Table S4). Thus, either low ODC1 expression or an AG/AA SNP genotype is associated with poor outcome in colorectal and lung cancer. Despite the differing role of ODC1 in neuroblastoma, these findings are consistent with our observations that the A allele is linked to lower ODC1 expression.

To determine if transcriptional regulators of ODC1 are differentially expressed in the different tissue types, the DoRothEA interactions dataset, available through OmniPath (https:/ / omnipathdb.org/, accessed 27 March 2021 through Bioconductor and RStudio), was explored. The transcription factors (TFs) MYC, MAX, MXD1, MXD4, CREB1 (cAMPresponsive element-binding protein 1), SP1 and WT1 (Wilms' tumor 1) were identified as regulators of ODC1. Expression of these TFs was explored using the MegaSampler module within the R2 Genomics Analysis and Visualization Platform. As seen in Figure S9A, significant differences in expression across the datasets were observed (one-way ANOVA, $p<0.001$ ). Pairwise comparisons showed that, as expected, MYCN was significantly overexpressed in neuroblastoma datasets compared with the adult tumor datasets, whereas the inverse was seen for MYC (Figure S9B). Interestingly, CREB1 was consistently expressed at higher levels in neuroblastoma compared to the other tissues, and WT1 was expressed at lower levels in neuroblastoma compared to most of the adult cancer cohorts. The expression of the other TFs in neuroblastoma compared to the adult cancers was variable. It is therefore possible that the differential SNP effects in the adult cancers, by comparison with neuroblastoma, may be due to differential expression of some of these ODC1-regulating TFs.

\section{Discussion}

Our results show that the A and G alleles of the ODC1 G316A promoter SNP differentially affect $O D C 1$ expression, as well as MYCN-mediated $O D C 1$ transactivation of the E-box region and MYCN oncogenic processes in neuroblastoma cells in vitro. Substituting the A for the G allele in isogenic cells is sufficient to reduce ODC1 mRNA and protein expression and cell proliferation. The underlying molecular mechanism revealed that the A allele has decreased affinity for $\mathrm{MYCN}$, indicating that the region surrounding the E-box is critical in modulating ODC1 transcriptional function. This is consistent with our previous findings showing a two-fold enrichment in MYCN binding in the region of the ODC1 promoter containing the G316A SNP in SK-N-BE(2)-C cells, which carry the GG genotype, compared with LAN-1 cells, which carry the AG genotype [13]. In addition, compared to cells containing constructs with the $A$ allele, the $G$ allele resulted in greater stimulation of $O D C 1$ promoter activity by MYCN. These results contrast to previous studies in adult cancers examining this polymorphism $[18,19]$. In colorectal cancer, studies found that c-MYC had the greatest stimulatory effect on promoters containing the A allele, and interestingly MAD1 was only effective at repressing $O D C 1$ promoter activity in promoters containing the A allele $[18,20]$. c-MYC and MXI1 proteins may selectively bind the A-allele in breast cancer, although this study utilized two genetically distinct cell lines rather than isogenic lines [19]. We did not investigate the effect of transcriptional repressors such as MAD1 and MXI1 that, like MYCN, form heterodimers with MAX and bind to E-box sites within the promoters of target genes. Given these previous findings we could speculate that they would also have a greater repressive effect on the $O D C 1$ promoters containing the $\mathrm{G}$ allele. Therefore, the impact of the genotype on $O D C 1$ expression in neuroblastoma may depend on the balance of MYCN levels and MAD1/MXI1 levels, and their binding with MAX. 
Previous studies have found an influence of the G316A SNP on DFMO response. Patients carrying two copies of the $\mathrm{G}$ allele had reduced risk of colorectal adenoma recurrence after treatment with DFMO and sulindac, and A allele carriers experienced less treatment-related benefit [24]. We found that Cas9 AG clones are more sensitive to DFMO compared to the parental GG line, which we might expect since the AG clones generate lower levels of ODC1, proliferate at a slower rate and therefore less DFMO is needed to completely block ODC1 protein in these cells. Our findings are in agreement with previous studies in prostate cancer, where, although the ODC1 SNP was not significantly associated with risk, the effect of long-term DFMO treatment on patients with AG/AA genotypes resulted in a reduced prostate volume [25].

Since DFMO is currently in clinical trials for neuroblastoma, our study highlights a subset of patients that may respond differentially to DFMO treatment. The G316A SNP was also studied previously in a DFMO trial involving 18 neuroblastoma patients with relapsed or refractory disease [26]. While there was a tendency towards improved survival for patients with the ODC1 GG genotype compared to any A allele following DFMO treatment, there was no significant difference, and the effect on urinary polyamines was also not significant. However, the primary aim of the study was to investigate the safety of DFMO in children and given the small number of patients enrolled and the fact that they had received many previous therapies, a large clinical trial is required to address the effect of the G316A SNP in response to DFMO therapy in neuroblastoma.

We demonstrated in our study cohort that the ODC SNP has prognostic significance in $M Y C N$ amplified and non-amplified neuroblastoma with the AA genotype indicative of a better prognosis. However, this finding did not significantly replicate in the blood-derived DNA in the large 4982 patient GWAS cohort. The rs2303615 genotype in this cohort was not directly measured in the tumor samples by quantitative PCR, but imputed from a genome-wide SNP array in the blood-derived DNA samples and the imputation statistics at rs2302615 were robust. To confirm the discrepancy was not due to the use of an admixed population, we restricted the analysis to Caucasians of European ancestry, but obtained similar results. We also explored the possibility of preferential somatic amplification of the $G$ allele, but saw no evidence for this in the subset of GWAS patients with paired tumor DNA evaluated by SNP arrays or next generation sequencing. It is well known that neuroblastoma patients show significant amounts of tumor-derived DNA in circulation, so we considered the possibility that somatic "contamination" influenced the blood-derived DNA genotyping results. However, we saw no evidence for this after carefully removing blood samples with a clear somatic DNA signature, and also not seeing the same protective effect of the AA genotype in the subjects without $M Y C N$ amplification. Finally, it is clear that the most important prognostic factor for any cancer patient is response to therapy received. We have shown that the survival rates for high-risk disease are improved in the GWAS cohort compared to the older cohort and thus is likely to have an impact on the influence of the SNP on survival. In addition, MYCN amplification status is a robust prognostic marker of outcome, but with the intensification of chemoradiotherapy, recent high-risk neuroblastoma trials showed no impact on outcome for patients whose tumors harbor MYCN amplification [27,28], unlike legacy high-risk trials in the past [29]. Since the GWAS cohort was accrued more recently, treatment era might be the best explanation for the discrepant results. Consistent with this, the outcomes in the study cohort are worse than those in the GWAS cohort overall, showing that the outcomes have improved between the timeframes of the two cohorts. Nevertheless, $M Y C N$ amplification remains an important risk stratification variable and it remains to be seen whether current therapeutic strategies directed towards polyamine depletion will be influenced by this ODC1 polymorphism.

Previous work has demonstrated a protective role for the 316A allele against the recurrence of colon polyps in clinical prevention trials, and the A allele significantly decreased the risk of developing sporadic breast cancer $[20,21,30]$. However, for breast cancer patients with established disease, the opposite has been found to be true where patients with one or two A alleles had a significantly lower 10-year survival compared 
to patients with the GG genotype, while colorectal cancer patients with an A allele had a median survival of $81 \%$ compared to $89 \%$ for GG $[18,19]$. Neither of these studies addressed whether ODC1 expression is directly altered by the SNP, although it is known that ODC1 levels are certainly increased compared to normal tissues in both breast and colon cancer $[7,31]$. The literature surrounding breast and colorectal cancer prognosis in relation to $O D C 1$ expression is not conclusive [8,32-35], whereas in neuroblastoma it is well established that high ODC1 mRNA expression is associated with poor outcome $[10,11,13]$.

Since there are surprisingly few studies looking at $O D C 1$ expression in adult cancers, we examined a publicly available database and showed that low ODC1 mRNA expression was prognostic of poor relapse-free survival in colorectal cancer. The finding in our own NSCLC cohort that low mRNA expression of ODC1 was also associated with worse outcome confirms an earlier report where similar low levels were associated with more aggressive lung tumors [36]. Overall, these data are in contrast with the neuroblastoma results and suggest a unique biology for this MYCN-driven pediatric malignancy where the AA genotype predicts a better outcome and where high ODC1 expression is consistently associated with poor outcome. Interestingly, upon examination of other known ODC1 transcriptional regulators, we found that CREB1 was expressed at higher levels in neuroblastoma compared to other adult tumor types, whereas WT1 was expressed at lower levels. These differences, together with other differences in the pattern of expression of ODC-regulating TFs in neuroblastoma versus other adult cancers, could have relevance in explaining the differential effects seen by the ODC1 SNP between neuroblastoma and adult cancers.

CREB1 is involved in tumorigenic processes such as proliferation, invasion and metastasis [37,38] and is overexpressed in many cancer types including neuroblastoma [39-41]. It has recently been identified as a direct target of miR-205 in neuroblastoma and colon cancer, with miR-205 playing a role in inhibiting CREB1 [42,43]. WT1 has been shown to be overexpressed and associated with poor outcome in several human tumors [44,45], although some reports suggest that WT1 is not associated with oncogenicity in neuroblastoma $[46,47]$. A recent study has found that WT1 expression is inversely correlated with MYCN expression in neuroblastoma, confirming our findings in this study, and whilst a mechanistic link is unclear, an association between high WT1 expression and poor outcome in non-MYCN amplified neuroblastomas was observed [48].

While the patient samples in our study cohort that expressed the highest levels of $O D C 1$ transcript exhibited a GG genotype, we were unable to show a significant difference in $O D C 1$ expression level in the neuroblastoma cohorts split by genotype. Thus, despite showing that the AG/GG genotypes lead to higher ODC1 transcript levels compared to the AA genotype in vitro, the difficulty in confirming this in primary tumor samples may be linked to a number of factors including: the relatively rare frequency of the A allele; tumor/stromal cell heterogeneity; and other determinants such as epigenetic modifications (at the level of DNA and/or chromatin) that can heavily affect ODC1 transcription. In addition, it is very difficult to measure ODC1 protein expression levels in tumor samples since ODC1 is a very low abundance protein and is highly regulated not only at the transcriptional level, but also by degradation. Interestingly, the SNP is embedded in a long $\mathrm{CpG}$ island in which $\mathrm{CpG}$ motifs are near each E-box, raising the possibility that the methylation status in this region may also influence the regulation of $O D C 1$ expression.

\section{Materials and Methods}

\subsection{Cell Culture and Reagents}

Tet $21 \mathrm{~N}$ cells, derived from the SHEP neuroblastoma cell line, stably express MYCN under the control of a tetracycline responsive promoter, where addition of tetracycline at $2 \mu \mathrm{g} / \mathrm{mL}$ represses expression of MYCN (Tet-off) [4]. Human neuroblastoma SK-N-BE(2)-C cells and Tet21N cells were maintained in Dulbecco's Modified Eagle's Medium (DMEM) (Life Technologies, Carlsbad, CA, USA) with 10\% fetal calf serum (FCS) (Life Technologies, 
Carlsbad, CA, USA) at $37^{\circ} \mathrm{C} / 5 \% \mathrm{CO}_{2}$. Their identities were verified by STR profiling (BMR Genomics, Padula, Italy) and cells were routinely mycoplasma tested.

\subsection{CRISPR-Cas9 Genome Editing}

Two specific single-guide RNAs (sgRNAs) targeting the G SNP were designed using the BlueHeron Guide RNA Target Design Tool (Blue Heron Biotech, Bothell, WA, USA), and CasOT 1.0 program (PKU Zebrafish Functional Genomics Group, Peking University, Beijing, China) [49]: sgRNA1: 5'-CGCCGGCCTGCGGAGACACG-3' and sgRNA2: $5^{\prime}$-CGGCGACCACGTGTCTCCGC-3'. The sgRNAs were cloned into the pCas-GuideEF1a-GFP vector (Origene, Rockville, MD, USA) and transfected into SK-N-BE(2)-C cells. Cleavage efficiencies were tested using GeneArt Genomic Cleavage Detection Kit (Thermo Fisher Scientific, Waltham, MA, USA) and sgRNA1 was selected for subsequent experiments. The absence of off-target activity was verified on the most probable off-target site by GeneArt Genomic Cleavage Detection Kit and confirmed by DNA sequencing. A donor template DNA was employed to mediate the A/G switch of the SNP by homologydirected repair (HDR). Donor DNA was amplified by PCR from NBL-S genomic DNA (primers: $5^{\prime}$-GTGCTATAAGTAGGGAGCG-3' ${ }^{\prime}$ and $5^{\prime}$-AAACTGGAAGGAAACTGAAG-3'). The obtained donor DNA was 683 bp-long with $351 \mathrm{bp}$ and $331 \mathrm{bp}$ homologous arms. SK-N-BE(2)-C cells were transfected using Lipofectamine 3000 (Thermo Fisher Scientific, Waltham, MA, USA), and isolated by serial dilutions $48 \mathrm{~h}$ later. Clonal cell lines were picked, expanded and characterized by RFLP analysis and DNA sequencing. Of the 81 screened clones, the calculated efficiency of successful heterozygous HDR-editing was $2.5 \%$, while $16 \%$ carried indel mutations.

\subsection{BrdU Cell Proliferation Assay}

Proliferation rates of CRISPR-edited SK-N-BE(2)-C clones were measured using bromodeoxyUridine (BrdU) incorporation (Cell Proliferation ELISA, BrdU (colorimetric), Roche, Basel, Switzerland), according to manufacturer's instructions. Cells were cultured for 7 days. $10 \mu \mathrm{M}$ BrdU was added and incorporation of BrdU quantified by ELISA $24 \mathrm{~h}$ later.

\subsection{ODC1 Expression in CRISPR-Edited Clones}

ODC1 expression in CRISPR-edited clones was determined by qRT-PCR and Western blot as previously described [50]. Primers are listed in Table S5. Rabbit monoclonal anti-Odc1 (Abcam, Cambridge, UK; ab126590) and rabbit monoclonal anti- $\beta$-Actin (SigmaAldrich, St. Louis, MO, USA; a2066) antibodies were used.

\subsection{Chromatin-Immunoprecipitation (ChIP)}

Standard ChIP assays were performed as previously described [50]. The analyzed region is shown in Figure S3A. Anti-Acetyl-Histone H3 (Merck, Rahway, NJ, USA; 06-599) antibody was used and primers are listed in Table S5.

\subsection{Clonogenic Assay}

Colony assays were performed as previously described [13]. SK-N-BE(2)-C cells were treated with 0-0.4 mM DFMO (Vinci Biochem, Florence, Italy). The total surface area occupied by the colonies within each well was determined using ImageJ software (version 1.51, NIH and LOCI, Madison, WI, USA).

\subsection{Electrophoretic Mobility Shift Assays (EMSA)}

SNP A/G DNA minimal probes, corresponding to region +301 to +334 of the ODC1 promoter including SNP A/G, were prepared from single strand synthetic oligonucleotides (Table S6). DNA probes used for cold competition assays were prepared from synthetic oligonucleotides containing the canonical wild type E-box using previously validated probes [51] and the mutated E-box (Table S6). Constructs are shown in Figure S3B. Comple- 
mentary sense and antisense oligonucleotides were annealed, double stranded probes were $5^{\prime}$ termini labelled with $\left[\gamma^{-32}\right.$ P]ATP using T4 Polynucleotide Kinase (New England Biolabs, MA, USA) and labelled probes were purified using QIAquick Nucleotide Removal Kit (Qiagen, Hilden, Germany). Nuclear protein extracts were prepared from SK-N-BE(2)-C and Tet21N cell lines as previously described [52]. Binding reactions were performed in EMSA-binding buffer using $100 \mathrm{fmol}$ of each labelled probe and $10 \mu \mathrm{g}$ of nuclear protein extract at $20^{\circ} \mathrm{C}$ for $35 \mathrm{~min}$. For competition experiments, 5-, 10- and 25-fold molar excess of wildtype or mutated unlabeled DNA probes were added. The reaction mixture was separated on $5 \%$ polyacrylamide gel then exposed to Kodak ${ }^{\circledR}$ BioMax ${ }^{\circledR}$ MS film (Kodak, Rochester, NY, USA) overnight at $-80^{\circ} \mathrm{C}$. The relative intensities of the bands (normalized for each specific free probe stain) were analyzed with Bio-Rad Quantity One ${ }^{\circledR}$ 1-D Analysis software (version 25.0, Bio-Rad Laboratories, Hercules, CA, USA).

\subsection{Luciferase Reporter Assay}

The ODC1 promoter region containing the SNP G316A (from +257 to +330 ) was amplified by nested PCR from SK-N-BE(2)-C (GG genotype) and NBL-S (AA genotype) genomic DNA. The sequences containing SNP G and SNP A were cloned into the luciferase pGL3 basic promoter vector (Promega, Fitchburg, WI, USA) (construct used is shown in Figure S3B). The Renilla-TK vector was used as an internal control. Firefly or Renilla luciferase activity was measured with the Dual Luciferase Assay kit (Promega).

\subsection{Cohort Descriptions}

Our study cohort is made up of neuroblastoma samples from the USA, Australia and Europe. The USA samples are from 183 patients enrolled between 1994-1998 by the Pediatric Oncology Group [53]. Australian samples are from 185 patients who were diagnosed in Australia and New Zealand between 1985-2000 [54]. DNA for the USA and Australian samples was isolated and RNA extracted as previously described $[53,55,56]$. The European samples are made up of 148 tumor RNA samples from the International Society of Pediatric Oncology European Neuroblastoma Group study (SIOPEN) [57], and 142 tumor RNA samples from the European Neuroblastoma Research Consortium (NRC), collected before 2012. DNA was amplified prior to genotyping as previously described [58]. RNA was available and of sufficient quality for expression analysis for 290 tumors.

GWAS cohort: Peripheral blood or uninvolved bone marrow DNA from 4892 neuroblastoma cases diagnosed up until 2016 in the USA and registered through the Children's Oncology Group (COG) was genotyped using Illumina HumanHap550 v1, HumanHap550 v3, HumanHap550 v3duo, Human610 Quad v1.0 and OmniExpress-24 SNP arrays, as described previously [59]. Genotypes for this multi-ethnic cohort were merged using PLINK [60] (v1.90p) and haplotypes inferred using SHAPEIT [61] (v2.r881). To impute genotypes across the genome, we utilized IMPUTE2 [62] (v2.3.2) with default parameters and $\mathrm{Ne}=20000$, along with a multi-population reference panel from the world-wide 1000 Genomes Project Phase 3. Imputed genotypes for rs2302615 were required to achieve an IMPUTE2 info quality score $>0.9$ and have inferred genotype probability $\geq 0.90$.

Lung Cancer cohort: DNA was extracted from fresh frozen surgically resected tumor tissue obtained from 366 patients with non-small cell lung cancer (NSCLC) who donated remnant tissue samples to The Prince Charles Hospital, Brisbane, Australia between 1992-2009. The cohort included 169 patients with adenocarcinoma, 161 patients with squamous cell carcinoma (SCC) and 36 patients with other histologies. The study was approved by the human research ethics committees at The Prince Charles Hospital (HREC EC9124, 17 October 2008) and Sydney Children's Hospital, part of the South Eastern Sydney Area Health Service (07/101, 22 May 2007-replaced by The University of New South Wales (HC12551, 16 October 2012). 


\subsection{ODC1 Expression in Neuroblastoma Patients}

For the USA samples of the study cohort, RNA was reverse-transcribed using MMLV reverse-transcriptase (Life Technologies). Gene expression levels were quantified by qRTPCR using TaqMan ${ }^{\circledR}$ Gene Expression Assays (Applied Biosystems ${ }^{\mathrm{TM}}$, Foster City, CA, USA) on a $96 \times 96$ Integrated Fluidics Circuit (BioMark HD System, Fluidigm, San Francisco, CA, USA). Data was normalized to a panel of control genes, HPRT1, GUSB, PPIA, HMBS and SDHA. Taqman assays used were ODC1 Hs00159739_m1, HPRT1 Hs99999909_m1, GUSB Hs99999908_m1, PPIA Hs99999904_m1, HMBS Hs00609296_g and SDHA Hs00188166_m1.

For the European samples of the study cohort, qRT-PCR based analysis of gene expression on a LightCycler480 (Roche) was performed as previously described [57]. ODC1 expression was measured using a previously reported TaqMan assay [10]. Measurement of Alu-Sq, HMBS, HPRT1, SDHA and UBC expression and normalization of ODC1 expression using the geometric mean expression levels of these five reference sequences were performed as previously described [58].

\subsection{SNP Genotyping}

RT-PCR was used to genotype the ODC1 promoter SNP G316A [14]. Primers and probes specific for either the $G$ or A allele were designed and supplied by Applied Biosystems Custom Taqman ${ }^{\circledR}$ SNP Genotyping Assay Service (Barcode 0039116973, Applied Biosystems, Foster City, CA, USA). The minor groove binder fluorogenic probes were labelled with FAM or VIC specific for the G and A alleles, respectively. Primer and probe sequences were: ODC-G316A-F 5'-CCGGGCACGTGTGC-3'; ODC-G316A-R 5'GAAGCGGCGCCTCAAG-3'; ODC-G probe 5'-CTGCGGAGACACG-3'; ODC-A probe 5'-CCTGCAGAGACACG-3'. 2x TaqMan Genotyping Master Mix (Applied Biosystems) and $40 \times$ ODC1 primer/probes mix was added to $10 \mathrm{ng}$ of genomic DNA. Reactions were subjected to a 10 -min denaturation at $95^{\circ} \mathrm{C}$, followed by 40 cycles of two-step PCR $\left(92{ }^{\circ} \mathrm{C}\right.$ for $15 \mathrm{~s}, 60^{\circ} \mathrm{C}$ for $1 \mathrm{~min}$ ). A post-read was immediately performed using the ABI Prism 7900 sequence detection system to determine endpoint fluorescence. PCR reactions were performed in duplicate and allelic discrimination was determined by laboratory personnel blinded to clinical data.

\subsection{Statistical Procedures}

Associations between clinical characteristics of patients and molecular characteristics of tumors were examined using Fisher's exact test. Associations with event-free survival (EFS) and overall survival (OS) were determined using Kaplan-Meier analyses and log-rank tests, or Cox regression for multivariate analyses, using SPSS version 24 (IBM, Armonk, NY, USA) as previously described [63]. For expression analyses, tumors were categorized as having high or low gene expression based on median cut-points, and a Cox model produced a $p$ value and a hazard ratio.

Experiments were repeated at least 3 times and the mean \pm standard error calculated. Differences between 2 groups were determined with an unpaired two-tailed Student's $t$-test. For 3 groups, one-way ANOVA tests were performed with correction according to Giesser-Greenhouse. To examine the influence of the SNP (A versus G) and MYCN expression (not expressed versus overexpressed) on promoter activity, a two-way ANOVA was conducted followed by Tukey's post-hoc tests for multiple pairwise comparisons. A probability level $p<0.05$ was considered to be statistically significant.

To identify transcriptional regulators of ODC1 expression, the DoRothEA interactions dataset, which is available through the OmniPath package and Bioconductor software in RStudio (version 1.2.1335, R Core Team, Vienna, Austria), was utilized. Only transcription factors with a high confidence level (rated ' $\mathrm{A}$ '), derived from the number of supporting evidences of a TF-interaction, were selected. These were then explored using the MegaSamper module in the R2 Analysis Genomics and Visualization platform where datasets that use the same chip (u133p2) and the same normalization method (MAS5.0) can be compared. A one-way ANOVA was used to detect significant differences across the datasets, and if a sig- 
nificant difference was detected Tukey's post-hoc tests for multiple pairwise comparisons were performed.

\section{Conclusions}

We have shown that the ODC1 G316A polymorphism is functionally important in childhood neuroblastoma. In particular, we found allele-specific regulation of this SNP by MYCN, in which this oncoprotein preferentially binds the $G$ allele over the A allele leading to a greater stimulatory effect on the $O D C 1$ promoter. These results in neuroblastoma contrast with those in adult cancers, and suggest tumor-specific regulation of $O D C 1$ by E-box transcription factors.

Supplementary Materials: The following are available online at https:/ /www.mdpi.com/article/10 $.3390 /$ cancers $13081807 / \mathrm{s} 1$, Table S1: Our study cohort is made up of samples from Europe, the USA and Australia, Table S2: Multivariate analyses for the different genotypes in the 839 patient study cohort (EFS data is available for 839 patients and OS data for 838 patients), and in the 4892 patient GWAS cohort (EFS and OS is available for all patients), Table S3: The number of each genotype in a non-small cell lung cancer cohort of 366 patients of mixed histologies (all), and in split cohorts of adenocarcinoma and squamous cell carcinoma, and the prognostic impact of these genotypes on outcome, Table S4: Multivariate analyses for the squamous cell carcinoma cohort (161 patients), Table S5: Primers used for qRT-PCR analysis of ODC1 expression (rows 1-4) and for ChIP (rows 5-16) in CRISPR-edited clones, Table S6: Probes used for EMSA assays, Figure S1: The ODC1 SNP at +316 is in intron 1 of the ODC1 transcript, and lies between 2 consensus E-box binding elements, Figure S2: Sequencing of the parental SK-N-BE(2)-C cells which are of GG genotype at the +316 SNP site, and the two AG clones generated by CRISPR-Cas9 technology, Figure S3: Schematic diagrams of the structure of the ODC1 gene and promoter, Figure S4: Quantification of the EMSA assays shown in Figure 2A, Figure S5: Survival analysis stratified by stage/risk group, age and $M Y C N$ amplification status, Figure S6: Survival analysis for the study cohort and the GWAS cohort, Figure S7: Separate survival analysis of the three distinct cohorts that were combined to make the study cohort, Figure S8: Survival analysis for high-risk patients, Figure S9: Expression of the transcriptional regulators of ODC1, as identified via the DoRothEA interactions dataset available in OmniPath, across the multiple tumor types, Figure S10: Uncropped WB of Figure 1C.

Author Contributions: Conceptualization, M.J.H., M.D.N. and M.H.; Data curation, L.D.G., S.P., M.J.H. and A.J.R.; Formal analysis, L.D.G., S.P., M.J.H., S.D.G., A.J.R., P.P., F.M.G., L.J.A., A.R. and T.V.M.; Funding acquisition, A.R., T.V.M., J.M.M., G.P., M.D.N. and M.H.; Investigation, L.D.G., S.P., M.J.H., S.D.G., A.J.R., P.P., J.M., E.V., G.M. and G.P.; Methodology, L.D.G., S.P., M.J.H., A.J.R., J.M., E.V., G.M., M.C., G.P., M.D.N. and M.H.; Project administration, G.P., M.D.N. and M.H.; Resources, M.J.H., J.B., G.S., A.R., T.V.M., J.V., F.S., R.V., J.K., A.E., R.N., R.L.S., G.P.T., K.F., Z.V., S.J.D., J.M.M., W.B.L., M.D.H., G.P., M.D.N. and M.H.; Supervision, G.P., M.D.N. and M.H.; Validation, L.D.G., S.P., A.J.R. and J.M.; Writing—original draft, L.D.G.; Writing—review and editing, L.D.G., S.P., M.J.H., S.D.G., J.M., G.S., T.V.M., J.V., F.S., R.V., A.E., R.N., R.L.S., G.P.T., S.J.D., J.M.M., W.B.L., G.M.M., D.S.Z., M.D.H., G.P., M.D.N. and M.H. All authors have read and agreed to the published version of the manuscript.

Funding: This study was supported by grants to G.M.M., M.D.N. and M.H. from the National Health and Medical Research Council (APP1132608), Cancer Council NSW (PG16-01), Cancer Institute NSW (12/TPG/1-13), grants from the Italian Association for Research on Cancer (AIRC) to G.P. (IG11400 and IG15182), grants from the Ghent University research fund to A.R. (BOF/01D02210) and the Research Foundation-Flanders to T.V.M. (FWO/1803115N), and grants from NIH to J.M.M. (R35 CA220500 and R01 CA124709). In addition this study was supported by the Annenberg Foundation, the Association Hubert Gouin Enfance et Cancer, the Association Enfants et Santé, Les Bagouz à Manon, Les amis de Claire. Funding was obtained from SiRIC/INCa (Grant INCa-DGOS-4654), as well as from the Fondation ARC pour la recherche sur le cancer.

Institutional Review Board Statement: The study was conducted according to the guidelines of the Declaration of Helsinki, and approved by the Institutional Review Board (or Ethics Committee) of the SYDNEY CHILDREN'S HOSPITAL, part of the SOUTH EASTERN SYDNEY AREA HEALTH SERVICE (SESAHS), (HREC 01-070, 25 September 2002, HREC 05/171, 24 August 2005, HREC 07 / 101 22 May 2007), GHENT UNIVERSITY (B670201111331, 07 March 2011) and the PRINCE CHARLES 
HOSPITAL (HREC EC9124, 17 October 2008). Ethical review and approval were waived for the GWAS study as it was deemed to be non-human subjects research by the CHOP IRB due to the fact that the samples were deidentified through the COG Biobanking study with no possibility of linking samples to subjects.

Informed Consent Statement: Informed consent was obtained from all subjects involved in the study.

Data Availability Statement: The data for the study cohort is available on request from the authors. The data for the GWAS cohort is available through the Database of Genotypes and Phenotypes (dbGaP) (Accession phs000124, https:/ / www.ncbi.nlm.nih.gov/projects/gap/cgi-bin/study.cgi? study_id=phs000124.v3.p1).

Acknowledgments: Children's Cancer Institute Australia is affiliated with UNSW Sydney and the Sydney Children's Hospitals Network. The results published here are in part based upon data generated by the Therapeutically Applicable Research to Generate Effective Treatments (https: // ocg.cancer.gov/programs/target) initiative, phs000467.

Conflicts of Interest: The authors declare no conflict of interest.

\section{References}

1. Cohn, S.L.; Tweddle, D.A. MYCN amplification remains prognostically strong 20 years after its "clinical debut". Eur. J. Cancer 2004, 40, 2639-2642. [CrossRef] [PubMed]

2. Thomas, T.; Thomas, T.J. Polyamine metabolism and cancer. J. Cell Mol. Med. 2003, 7, 113-126. [CrossRef] [PubMed]

3. Bello-Fernandez, C.; Packham, G.; Cleveland, J.L. The ornithine decarboxylase gene is a transcriptional target of c-Myc. Proc. Natl. Acad. Sci. USA 1993, 90, 7804-7808. [CrossRef] [PubMed]

4. Lutz, W.; Stöhr, M.; Schürmann, J.; Wenzel, A.; Löhr, A.; Schwab, M. Conditional expression of N-MYC in human neuroblastoma cells increases expression of alpha-prothymosin and ornithine decarboxylase and accelerates progression into S-phase early after mitogenic stimulation of quiescent cells. Oncogene 1996, 13, 803-812.

5. Gerner, E.W.; Meyskens, F.L., Jr. Polyamines and cancer: Old molecules, new understanding. Nat. Rev. Cancer 2004, 4, 781-792. [CrossRef]

6. Tian, H.; Huang, Q.; Li, L.; Liu, X.-X.; Zhang, Y. Gene expression of ornithine decarboxylase in lung cancers and its clinical significance. Acta Biochim. Biophys. Sin. 2006, 38, 639-645. [CrossRef]

7. $\quad$ Deng, W.; Jiang, X.; Mei, Y.; Sun, J.; Ma, R.; Liu, X.; Sun, H.; Tian, H.; Sun, X. Role of ornithine decarboxylase in breast cancer. Acta Biochim. Biophys. Sin. 2008, 40, 235-243. [CrossRef]

8. Hoshino, Y.; Terashima, S.; Teranishi, Y.; Terashima, M.; Kogure, M.; Saitoh, T.; Osuka, F.; Kashimura, S.; Saze, Z.; Gotoh, M. Ornithine decarboxylase activity as a prognostic marker for colorectal cancer. Fukushima J. Med. Sci. 2007, 53, 1-9. [CrossRef] [PubMed]

9. Mohan, R.R.; Challa, A.; Gupta, S.; Bostwick, D.G.; Ahmad, N.; Agarwal, R.; Marengo, S.R.; Amini, S.B.; Paras, F.; MacLennan, G.T.; et al. Overexpression of ornithine decarboxylase in prostate cancer and prostatic fluid in humans. Clin. Cancer Res. 1999, 5, 143-147. [PubMed]

10. Hogarty, M.D.; Norris, M.D.; Davis, K.; Liu, X.; Evageliou, N.F.; Hayes, C.S.; Pawel, B.; Guo, R.; Zhao, H.; Sekyere, E.; et al. ODC1 is a critical determinant of MYCN oncogenesis and a therapeutic target in neuroblastoma. Cancer Res. 2008, 68, 9735-9745. [CrossRef]

11. Geerts, D.; Koster, J.; Albert, D.; Koomoa, D.L.; Feith, D.J.; Pegg, A.E.; Caron, H.; Bachmann, A. The polyamine metabolism genes ornithine decarboxylase and antizyme 2 predict aggressive behavior in neuroblastomas with and without MYCN amplification. Int. J. Cancer 2010, 126, 2012-2024. [PubMed]

12. Evageliou, N.F.; Haber, M.; Vu, A.; Laetsch, T.W.; Murray, J.; Gamble, L.D.; Cheng, N.C.; Liu, K.; Reese, M.; Corrigan, K.A.; et al. Polyamine antagonist therapies inhibit neuroblastoma initiation and progression. Clin. Cancer Res. 2016, 22, 4391-4404. [CrossRef] [PubMed]

13. Gamble, L.D.; Purgato, S.; Murray, J.; Xiao, L.; Yu, D.M.T.; Hanssen, K.M.; Giorgi, F.M.; Carter, D.R.; Gifford, A.J.; Valli, E.; et al. Inhibition of polyamine synthesis and uptake reduces tumor progression and prolongs survival in mouse models of neuroblastoma. Sci. Transl. Med. 2019, 11, 1099. [CrossRef] [PubMed]

14. Guo, Y.; Harris, R.B.; Rosson, D.; Boorman, D.; O’Brien, T.G. Functional analysis of human ornithine decarboxylase alleles. Cancer Res. 2000, 60, 6314-6317. [PubMed]

15. Walhout, A.J.M.; Gubbels, M.-J.; Bernards, R.; Van Der Vliet, P.C.; Timmers, H.T.M. C-Myc/Max heterodimers bind cooperatively to the e-box sequences located in the first intron of the rat ornithine decarboxylase (ODC) gene. Nucleic Acids Res. 1997, 25, 1493-1501. [CrossRef]

16. Solomon, D.L.C.; Amati, B.; Land, H. Distinct DNA binding preferences for the c-Myc/Max and Max/Max dimers. Nucleic Acids Res. 1993, 21, 5372-5376. [CrossRef] [PubMed]

17. Walhout, A.; Van Der Vliet, P.C.; Timmers, H. Sequences flanking the E-box contribute to cooperative binding by c-Myc/Max heterodimers to adjacent binding sites. Biochim. Biophys. Acta Gene Struct. Expr. 1998, 1397, 189-201. [CrossRef] 
18. Zell, J.A.; Ziogas, A.; Ignatenko, N.; Honda, J.; Qu, N.; Bobbs, A.S.; Neuhausen, S.L.; Gerner, E.W.; Anton-Culver, H. Associations of a polymorphism in the ornithine decarboxylase gene with colorectal cancer survival. Clin. Cancer Res. 2009, 15, $6208-6216$. [CrossRef]

19. Xu, L.; Long, J.; Wang, P.; Liu, K.; Mai, L.; Guo, Y. Association between the ornithine decarboxylase G316A polymorphism and breast cancer survival. Oncol. Lett. 2015, 10, 485-491. [CrossRef]

20. Martínez, M.E.; O’Brien, T.G.; Fultz, K.E.; Babbar, N.; Yerushalmi, H.; Qu, N.; Guo, Y.; Boorman, D.; Einspahr, J.; Alberts, D.S.; et al . Pronounced reduction in adenoma recurrence associated with aspirin use and a polymorphism in the ornithine decarboxylase gene. Proc. Natl. Acad. Sci. USA 2003, 100, 7859-7864. [CrossRef]

21. Hubner, R.A.; Muir, K.R.; Liu, J.-F.; Logan, R.F.; Grainge, M.J.; Houlston, R.S. Ornithine decarboxylase G316A genotype is prognostic for colorectal adenoma recurrence and predicts efficacy of aspirin chemoprevention. Clin. Cancer Res. 2008, 14, 2303-2309. [CrossRef]

22. Willems, E.; Leyns, L.; Vandesompele, J. Standardization of real-time PCR gene expression data from independent biological replicates. Anal. Biochem. 2008, 379, 127-129. [CrossRef]

23. Győrffy, B.; Surowiak, P.; Budczies, J.; Lánczky, A. Online survival analysis software to assess the prognostic value of biomarkers using transcriptomic data in non-small-cell lung cancer. PLoS ONE 2013, 8, e82241. [CrossRef]

24. Zell, J.A.; McLaren, C.E.; Chen, W.-P.; Thompson, P.A.; Gerner, E.W.; Meyskens, F.L. Ornithine decarboxylase-1 polymorphism, chemoprevention with eflornithine and sulindac, and outcomes among colorectal adenoma patients. J. Natl. Cancer Inst. 2010, 102, 1513-1516. [CrossRef]

25. Meyskens, F.L., Jr.; Simoneau, A.R.; Gerner, E.W. Chemoprevention of prostate cancer with the polyamine synthesis inhibitor difluoromethylornithine. Recent results in cancer research. Fortschr. Krebsforsch. Prog. Rech. Cancer 2014, 202, 115-120.

26. Sholler, S.G.L.; Gerner, E.W.; Bergendahl, G.; MacArthur, R.B.; VanderWerff, A.; Ashikaga, T.; Ferguson, W.; Moriya, A. A phase I trial of DFMO targeting polyamine addiction in patients with relapsed/refractory neuroblastoma. PLoS ONE 2015, 10, e0127246. [CrossRef] [PubMed]

27. Park, J.R.; Kreissman, S.G.; London, W.B.; Naranjo, A.; Cohn, S.L.; Hogarty, M.D.; Kamagutchi, L. Effect of tandem autologous stem cell transplant vs single transplant on event-free survival in patients with high-risk neuroblastoma: A randomized clinical trial. JAMA 2019, 322, 746-755. [CrossRef] [PubMed]

28. Ladenstein, R.; Pötschger, U.; Pearson, A.D.J.; Brock, P.; Luksch, R.; Castel, V.; Yaniv, I.; Papadakis, V.; Laureys, G.; Malis, J.; et al. Busulfan and melphalan versus carboplatin, etoposide, and melphalan as high-dose chemotherapy for high-risk neuroblastoma (HR-NBL1/SIOPEN): An international, randomised, multi-arm, open-label, phase 3 trial. Lancet Oncol. 2017, 18, 500-514. [CrossRef]

29. Schneiderman, J.; London, W.B.; Brodeur, G.M.; Castleberry, R.P.; Look, A.T.; Cohn, S.L. Clinical significance of MYCN amplification and ploidy in favorable-stage neuroblastoma: A report from the Children's Oncology Group. J. Clin. Oncol. 2008, 26, 913-918. [CrossRef] [PubMed]

30. O'Connell, B.C.; Cheung, A.F.; Simkevich, C.P.; Tam, W.; Ren, X.; Mateyak, M.K.; Sedivy, J.M. A large scale genetic analysis of c-Myc-regulated gene expression patterns. J. Biol. Chem. 2003, 278, 12563-12573. [CrossRef]

31. Linsalata, M.; Russo, F.; Cavallini, A.; Berloco, P.; Di Leo, A. Polyamines, diamine oxidase, and ornithine decarboxylase activity in colorectal cancer and in normal surrounding mucosa. Dis. Colon Rectum 1993, 36, 662-667. [CrossRef]

32. Matsubara, N.; Hietala, A.O.; Gilmour, S.K.; Yum, K.Y.; Litwin, S.; Watts, P.; Brennan. O'Brien Association between high levels of ornithine decarboxylase activity and favorable prognosis in human colorectal carcinoma. Clin. Cancer Res. 1995, 1, 665-671.

33. Manni, A.; Mauger, D.; Gimotty, P.; Badger, B. Prognostic influence on survival of increased ornithine decarboxylase activity in human breast cancer. Clin. Cancer Res. 1996, 2, 1901-1906.

34. Cañizares, F.; Salinas, J.; Heras, M.D.L.; Diaz, J.; Tovar, I.; Martinez, P.; Peñafiel, R. Prognostic value of ornithine decarboxylase and polyamines in human breast cancer: Correlation with clinicopathologic parameters. Clin. Cancer Res. 1999, 5, $2035-2041$.

35. Love, R.R.; Astrow, S.H.; Cheeks, A.M.; Havighurst, T.C. Ornithine decarboxylase (ODC) as a prognostic factor in operable breast cancer. Breast Cancer Res. Treat. 2003, 79, 329-334. [CrossRef] [PubMed]

36. Grimminger, P.P.; Schneider, P.M.; Metzger, R.; Vallböhmer, D.; Danenberg, K.D.; Danenberg, P.V.; Hölscher, A.H.; Brabender, J. Ornithine decarboxylase mRNA expression in curatively resected non-small-cell lung cancer. Clin. Lung Cancer 2010, 11, 114-119. [CrossRef] [PubMed]

37. Sakamoto, K.M.; Frank, D.A. CREB in the pathophysiology of cancer: Implications for targeting transcription factors for cancer therapy. Clin. Cancer Res. 2009, 15, 2583-2587. [CrossRef] [PubMed]

38. Shukla, A.; Bosenberg, M.W.; MacPherson, M.B.; Butnor, K.J.; Heintz, N.H.; Pass, H.I.; Carbone, M.; Testa, J.R.; Mossman, B.T. Activated cAMP response element binding protein is overexpressed in human mesotheliomas and inhibits apoptosis. Am. J. Pathol. 2009, 175, 2197-2206. [CrossRef] [PubMed]

39. Ni, Y.; Zhou, Y.; Zhou, M.; Zhang, L. Akt and cAMP response element binding protein mediate 17beta-estradiol regulation of glucose transporter 3 expression in human SH-SY5Y neuroblastoma cell line. Neurosci. Lett. 2015, 604, 58-63. [CrossRef]

40. Zamarbide, M.; Etayo-Labiano, I.; Ricobaraza, A.; Martínez-Pinilla, E.; Aymerich, M.S.; Lanciego, J.L.; Pérez-Mediavilla, A.; Franco, R. GPR40 activation leads to CREB and ERK phosphorylation in primary cultures of neurons from the mouse CNS and in human neuroblastoma cells. Hippocampus 2014, 24, 733-739. [CrossRef] [PubMed] 
41. Sapio, L.; Salzillo, A.; Ragone, A.; Illiano, M.; Spina, A.; Naviglio, S. Targeting CREB in cancer therapy: A key candidate or one of many? An update. Cancers 2020, 12, 3166. [CrossRef] [PubMed]

42. Chen, S.; Jin, L.; Nie, S.; Han, L.; Lu, N.; Zhou, Y. miR-205 inhibits neuroblastoma growth by targeting cAMP-responsive element-binding protein. Oncol. Res. 2018, 26, 445-455. [CrossRef] [PubMed]

43. Li, P.; Xue, W.-J.; Feng, Y.; Mao, Q.-S. MicroRNA-205 functions as a tumor suppressor in colorectal cancer by targeting cAMP responsive element binding protein 1 (CREB1). Am. J. Transl. Res. 2015, 7, 2053-2059. [PubMed]

44. Inoue, K.; Sugiyama, H.; Ogawa, H.; Nakagawa, M.; Yamagami, T.; Miwa, H.; Kita, K.; Hiraoka, A.; Masaoka, T.; Nasu, K. WT1 as a new prognostic factor and a new marker for the detection of minimal residual disease in acute leukemia. Blood 1994, 84, 3071-3079. [CrossRef] [PubMed]

45. Miyoshi, Y.; Ando, A.; Egawa, C.; Taguchi, T.; Tamaki, Y.; Tamaki, H.; Sugiyama, H.; Noguchi, S. High expression of Wilms' tumor suppressor gene predicts poor prognosis in breast cancer patients. Clin. Cancer Res. 2002, 8, 1167-1171.

46. Dennis, S.L.; Manji, S.S.; Carrington, D.P.; Scarcella, D.L.; Ashley, D.M.; Smith, P.J.; Algar, E.M. Expression and mutation analysis of the Wilms' tumor 1 gene in human neural tumors. Int. J. Cancer 2002, 97, 713-715. [CrossRef]

47. Wang, J.; Oue, T.; Uehara, S.; Yamanaka, H.; Oji, Y.; Fukuzawa, M. The role of WT1 gene in neuroblastoma. J. Pediatr. Surg. 2011, 46, 326-331. [CrossRef]

48. Masserot, C.; Liu, Q.; Nguyen, E.; Gattolliat, C.-H.; Valteau-Couanet, D.; Bénard, J.; Huber, C.; Ségal-Bendirdjian, E. WT1 expression is inversely correlated with MYCN amplification or expression and associated with poor survival in non-MYCNamplified neuroblastoma. Mol. Oncol. 2015, 10, 240-252. [CrossRef] [PubMed]

49. Xiao, A.; Cheng, Z.; Kong, L.; Zhu, Z.; Lin, S.; Gao, G.; Zhang, B. CasOT: A genome-wide Cas9/gRNA off-target searching tool. Bioinformatics 2014, 30, 1180-1182. [CrossRef]

50. Iraci, N.; Diolaiti, D.; Papa, A.; Porro, A.; Valli, E.; Gherardi, S.; Smith, A.; Diollin, P. A SP1/MIZ1/MYCN repression complex recruits HDAC1 at the TRKA and p75NTR promoters and affects neuroblastoma malignancy by inhibiting the cell response to NGF. Cancer Res. 2011, 71, 404-412. [CrossRef]

51. Perini, G.; Diolaiti, D.; Porro, A.; Della Valle, G. In vivo transcriptional regulation of N-Myc target genes is controlled by E-box methylation. Proc. Natl. Acad. Sci. USA 2005, 102, 12117-12122. [CrossRef]

52. Dignam, J.D.; Lebovitz, R.M.; Roeder, R.G. Accurate transcription initiation by RNA polymerase II in a soluble extract from isolated mammalian nuclei. Nucleic Acids Res. 1983, 11, 1475-1489. [CrossRef] [PubMed]

53. Haber, M.; Smith, J.; Bordow, S.B.; Flemming, C.; Cohn, S.L.; London, W.B.; Marshall, G.M.; Norris, M.D. Association of high-level MRP1 expression with poor clinical outcome in a large prospective study of primary neuroblastoma. J. Clin. Oncol. 2006, 24, 1546-1553. [CrossRef] [PubMed]

54. Pajic, M.; Murray, J.; Marshall, G.M.; Cole, S.P.; Norris, M.D.; Haber, M. ABCC1 G2012T single nucleotide polymorphism is associated with patient outcome in primary neuroblastoma and altered stability of the ABCC1 gene transcript. Pharm. Genom. 2011, 21, 270-279. [CrossRef]

55. Bordow, S.B.; Haber, M.; Madafiglio, J.; Cheung, B.; Marshall, G.M.; Norris, M.D. Expression of the multidrug resistance-associated protein (MRP) gene correlates with amplification and overexpression of the $\mathrm{N}$-myc oncogene in childhood neuroblastoma. Cancer Res. 1994, 54, 5036-5040.

56. Norris, M.D.; Haber, M.; Gilbert, J.; Kavallaris, M.; Marshall, G.M.; Stewart, B.W. N-myc gene amplification in neuroblastoma determined by the polymerase chain reaction. Prog. Clin. Biol. Res. 1994, 385, 27-33.

57. Vermeulen, J.; De Preter, K.; Naranjo, A.; Vercruysse, L.; Van Roy, N.; Hellemans, J.; Swerts, K.; Bravo, S.; Scaruffi, P.; Tonini, G.P.; et al. Predicting outcomes for children with neuroblastoma using a multigene-expression signature: A retrospective SIOPEN/COG/GPOH study. Lancet Oncol. 2009, 10, 663-671. [CrossRef]

58. Rihani, A.; De Wilde, B.; Zeka, F.; Laureys, G.; Francotte, N.; Tonini, G.P.; Coco, S.; Versteeg, R.; Noguera, R.; Schulte, J.H.; et al. CASP8 SNP D302H (rs1045485) is associated with worse survival in MYCN-amplified neuroblastoma patients. PLoS ONE 2014, 9, e114696. [CrossRef]

59. Diskin, S.J.; Capasso, M.; Schnepp, R.W.; Cole, A.K.; Attiyeh, E.F.; Hou, C.; Diamond, M.; Carpenter, E.L.; Winter, C.; Lee, H.; et al. Common variation at 6q16 within HACE1 and LIN28B influences susceptibility to neuroblastoma. Nat. Genet. 2012, 44, 1126-1130. [CrossRef] [PubMed]

60. Purcell, S.; Neale, B.; Todd-Brown, K.; Thomas, L.; Ferreira, M.A.; Bender, D.; Maller, J.; Sklar, P.; de Bakker, P.I.; Daly, M.J.; et al. PLINK: A tool set for whole-genome association and population-based linkage analyses. Am. J. Hum. Genet. 2007, 81, 559-575. [CrossRef]

61. Delaneau, O.; Marchini, J.; Zagury, J.-F. A linear complexity phasing method for thousands of genomes. Nat. Methods 2011, 9, 179-181. [CrossRef] [PubMed]

62. Howie, B.N.; Donnelly, P.; Marchini, J. A flexible and accurate genotype imputation method for the next generation of genomewide association studies. PLoS Genet. 2009, 5, e1000529. [CrossRef] [PubMed]

63. Henderson, M.J.; Haber, M.; Porro, A.; Munoz, M.A.; Iraci, N.; Xue, C.; Murray, J.; Flemming, C.L.; Smith, J.; Fletcher, J.I.; et al. ABCC multidrug transporters in childhood neuroblastoma: Clinical and biological effects independent of cytotoxic drug efflux. J. Natl. Cancer Inst. 2011, 103, 1236-1251. [CrossRef] [PubMed] 